\title{
Using Semantic Technology to Model Persona for Adaptable Agents
}

\author{
Johannes Nguyen, Thomas Farrenkopf, \\ Michael Guckert \\ Kompetenzzentrum für Informationstechnologie \\ Technische Hochschule Mittelhessen \\ 61169, Friedberg, Germany \\ \{johannes.nguyen,thomas.farrenkopf, \\ michael.guckert\}@mnd.thm.de
}

\section{KEYWORDS}

Adaptable Agents, Persona, Agent Modelling, Semantic Technologies

\begin{abstract}
In state of the art research a growing interest in the application of agent models for the simulation of road traffic can be observed. Software agents are particularly suitable for the representation of travellers and their goal-oriented behaviour. Although numerous applications based on these types of models are already available, the options for modelling and calibration of the agents as goal-oriented individuals are either simplified to aggregated parameters or associated with overly complex and opaque implementation details. This makes it difficult to reuse available simulation models. In this paper, we demonstrate how the combination of persona models together with semantic methods can be applied to achieve a well-structured agent model that allows for improved reusability.
\end{abstract}

\section{INTRODUCTION}

Computer-based simulation is an accepted means for researching transportation questions, which has been used as early as the 1970s [1], [2]. The number of existing simulators is significant, with each of the tools focusing on different aspects of the transport system and differing in the underlying methods. There is a variety of simulators that range from more general purpose applications (e.g. [3], [4], [5], [6], [7]) to systems designed for specific research questions (e.g. [8], [9], [10]). In practical research on transportation, researchers are faced with the issue of finding appropriate simulators. [7] have described that even though general purpose applications such as MATSim [5] and SUMO [4] offer a lot of potential for reusability and sharing of common traffic concepts (e.g. modelling of road network, vehicles, traffic flow), in many cases researchers have instead implemented their own simulation models from scratch. A reason for this may be that customisation options in available simulators are either too limited or too complex to be implemented. This is the case when customisation requires advanced programming or a deep understanding of the underlying system. A structured

\author{
Simon T. Powers, Neil Urquhart \\ School of Computing \\ Edinburgh Napier University \\ EH10 5DT, Edinburgh, United Kingdom \\ \{s.powers,n.urquhart\}@napier.ac.uk
}

design with a clear separation of concerns (see [11]) for modelling software agents using persona models and semantic methods can help to improve reusability of simulation models and reduce complexity for customisation.

This paper is organised as follows: The following sections provides a short introduction into the theoretical background of persona models, which are usually applied to areas in which focus lies on user-centricity such as Human-Computer interaction (HCI) or marketing. Furthermore, an overview of the semantic instruments used in this work is given, namely ontologies implemented in OWL (Web Ontology Language) [12] and SWRL (Semantic Web Rule Language)[13]. Following this, we discuss related work. We then present a modelling method that allows for less complex customisation using the concepts of persona models and ontologies. As proof of concept, we perform simulation of two example scenarios using the AGADE Traffic simulator [14]. The scenarios fundamentally differ in types of mobility, which is often the case when specific research questions at hand deviate from the main focus of available simulators. Thus, we demonstrate how customisation or extensions to the model can be implemented with the proposed method. Finally, summary and conclusions are given as well as indications for future work.

\section{PRELIMINARIES}

The following section briefly introduces background knowledge on the concept of persona models and semantic methods.

\section{Persona Models}

Persona models are an instrument for analysing and modelling groups of individuals sharing similar behaviour. They are often applied in the field of $\mathrm{Hu}-$ man-Computer interaction (HCI) and for marketing purposes. In practical applications persona are usually created with segmentation or clustering methods based on collected customer or user data. [15] has discussed the origins of persona models as an approach to goaloriented software design. Reference is given to Cooper's definition of persona models as "a precise description 
of [a] user and what he wishes to accomplish" (see [16], p.123). A more detailed description is given by [17] who describe persona as "fictional, detailed archetypical characters that represent distinct groupings of behaviours, goals and motivations observed and identified during the research phase". It can be summarised that persona are fictional characters representing groups of individuals. They are identified by a unique name and carry additional descriptive information of relevance for the perspective that is to be modelled, e.g. appearance, private background, preferences, habits and goals in order to make a group of individuals more comprehensible and manageable and to convey their personality and motivations.

\section{Ontologies and Rules}

Ontologies are an expressive tool to model a domain in machine readable form and provide an explicit, shared specification of a conceptualisation [18]. Ontologies typically consist of a taxonomy of concepts each with properties and relations. OWL (Web Ontology Language) is a standardised implementation of a description logic based ontology language [12]. As description logic is object centered, formulation of simple if-then rules is limited. These rules can be expressed using Semantic Web Rule Language (SWRL). SWRL is also standardised by W3C. Inference engines derive knowledge by evaluating OWL and SWRL expressions.

\section{RELATED WORK}

We have reviewed a wide range of available traffic simulators in detail (inter alia [3], [5], [19], [8], [20], [6], [7], [21], [22], [23]). In particular, the AgentPolis approach stands out as it also reflects on the shortcomings of reusability in available simulation models. AgentPolis is a fully agent based traffic simulator that focuses on the simulation of interaction-rich transport scenarios [24], [7]. For example, simulation of on-demand mobility services (e.g ridesharing) requires interaction between service providers and customers but numerous other forms of interaction between travellers are possible. Despite the fact that general purpose traffic simulators such as MATSim and SUMO provide a variety of modelling concepts (e.g. road network, vehicles, traffic flow), the authors of AgentPolis identified the gap of transport scenarios with significant interaction between travellers and their immediate surrounding. The authors of AgentPolis concluded that similarities between simulation models have not been exploited sufficiently due to existing tools not taking into account the multiagent nature of interaction-rich transport systems. Reference is given to work in which model-specific simulation tools have been developed from scratch (see [10], [25], [9]). AgentPolis addresses these deficiencies and provides a set of abstractions, code libraries and software tools for building simulation models [7]. While focus of the project was on the modelling of interactionrich transport systems, a technical solution has also been implemented to facilitate the reuse of common transportation concepts. For this purpose, AgentPolis integrates a modelling abstraction ontology. The theoretical concept of this component is to separate defined modelling abstractions from implementations of specific modelling elements. It uses an ontology in order to define more general concepts of multi-agent systems. This approach results in a tailored structure for object-oriented programming that simplifies extending the simulation models for research-specific scenarios.

In this paper, we revisit this idea of reusable modelling concepts using ontologies in traffic simulations and further expand on the modelling capabilities of semantic methods. Furthermore, we will go one step further and place the individual and its decision-making at the center of attention in our modelling rather than solely defining general modelling abstractions in the ontology for common transportation concepts such as traffic lights, etc.

\section{MODELLING}

The application of agent-based models for simulating road traffic is an established method. Traffic is an emergent phenomenon in which global system behaviour is determined by a large set of individuals, each with their own goals and preferences. As [26] describe, software agents are closed computer systems that are situated in some environment, and that are capable of autonomous action in this environment in order to meet their designed objectives. This autonomous and goal-oriented behaviour also applies to travellers in the real world which is why software agents are particularly suitable for representing travellers in computer-based simulation models. The modelling of these individuals and their decision-making behaviour is often complex, and closely depends to the research question at hand. As a result, agent behaviour needs to be adjusted. For example, choice of transport mode in sightseeing scenarios differs from the choice in everyday commuting to work as travellers value time differently. It is precisely these adjustments in agent modelling that transportation researchers have to implement in order to be able to properly simulate their research scenarios in the first place. In this context, various researchers are repeatedly confronted with difficulties, as options for modelling and calibration of the agents are either simplified to aggregated parameters, or are associated with complex programming that often requires a deep understanding of the underlying software architecture. The problem does not only relate to researchers with a background in computing science, but also to those who would rather deal with traffic engineering issues exclusively. Consequently, it can be anticipated that these researchers will be overburdened when customising existing models, which is why new ontological concepts are needed to simplify this process. Otherwise, these researchers will start to develop their own simulation models from scratch as illustrated by [7].

As agent modelling essentially depends on the scenario being investigated, agents are usually modelled specifically for one particular scenario. For flexible reuse of agents in different scenarios, we need methods that en- 
able generalisation of agent behaviour. A similar problem can be observed in general problem solving which is a subcategory of artificial intelligence [27]. [28] analysed implementations of domain-specific problem solving, in order to identify abstraction patterns that can define different methods of general problem solving. These patterns have served as the basis for numerous subsequent research. Particularly, the CommonKADS project is one of the outcomes [29]. The project created its own abstraction patterns for general problem solving and also expanded on concepts of knowledge engineering. Based on this, [27] describe the expertise of a system as the combination of knowledge about the contexts of the observation subject at hand and the ability to draw conclusions. An example is given of the knowledge acquisition process for building an domain-specific problem solver that performs fault detection on bicycles: (1) First, a mechanic that specialises in bicycles is interviewed about his working methods. (2) In addition, the same mechanic is observed while at work in order to also capture implicit knowledge that cannot be expressed and described with words and that is needed for such a diagnosis. (3) Furthermore, documents such as repair manuals or measurement tables can be included. Collected knowledge can be merged into a unitary model of expertise. [27] point out that different types of knowledge are involved. More particularly, knowledge on the assembly of bicycles, about the mechanics, as well as knowledge about possible faults and their causes, and knowledge about the procedure for recognising and repairing faults. The CommonKADS project has defined a layered model for distinguishing the different types of knowledge (see figure 1). The lowest layer describes Domain Knowledge. In this layer, domain-specific concepts and simple relations are defined. Considering the example of fault detection for bicycles, information on this layer may include what a bicycle is, which parts it consists of, which possible faults may occur, as well as possible causes of faults and corrective measures. Inference Knowledge is located in the layer above. This layer contains information about the logical contexts of the concepts defined in the domain knowledge. Based on this, conclusions can be drawn using various methods and algorithms. Finally, at the top layer there is Task Knowledge in which information from the lower levels is brought together in order to perform decision-making and determine actions.

We now propose an architecture analogous to the CommonKADS knowledge structure as knowledge base for adaptable agents in traffic simulations (see Figure 2). This knowledge base is implemented by means of OWL ontologies extended with SWRL rules. We distinguish between two types of domain knowledge: travel and activity information. Concepts of the first type of domain knowledge are relevant for traffic related aspects such as mode options. To facilitate reusability, they are encoded in a separate ontology which we call the travel ontology. The travel ontology exclusively contains knowledge on common traffic concepts for example transportation modes, road signs, etc. The sec-

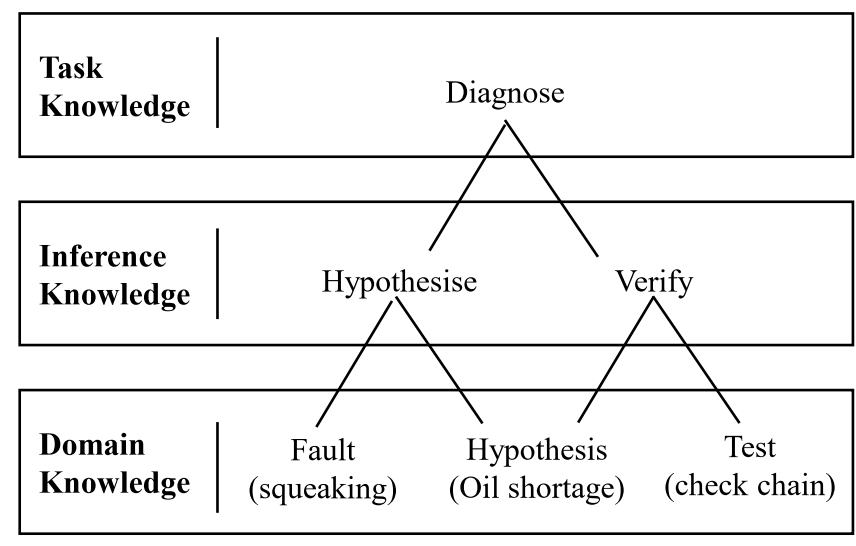

Fig. 1. CommonKADS: Types of Knowledge (see [20],[22]).

ond type of domain knowledge can be referred to as activity information and extends the knowledge of the agents by concepts that are relevant to model researchquestion-specific activity. For example, when simulating a sightseeing scenario agents require completely different activity information compared to a grocery shopping scenario. This method allows for a flexible extension of agent knowledge. Agents are not bound to one type of activity information, but may also integrate several activity ontologies for broader decision-making and simulation of more complex scenarios. Regarding the layer of inference knowledge, all ontologies containing travel or activity related information are consolidated (imported) into a central person ontology. This ontology contains information about person-specific concepts such as census properties. This enables the implementation of decision-making in various domains using only one software agent. The idea matches the individual in the real world, that is constantly required to make multi-criteria decisions based on preferences from various aspects in life. The defined concepts in the domain knowledge can be used to formulate a set of logic based inference rules that enables the application of computer based reasoners. By employing these established reasoning mechanisms, we use census information as input data to infer domain-specific preferences that can be used as criteria for agent decision making. For example, in a grocery shopping scenario travellers have to make a decision as to which supermarket they want to approach. This decision not only depends on traffic-related preferences (transport mode, shortest distance, etc.) but also on personal food preferences. This reflects different domains of knowledge. Travellers who particularly value organic and sustainable products would possibly be willing to travel to a specialist store for organic food even if the distance is a bit longer. For determining these preferences, rules can be defined according to the following scheme:

$$
\begin{aligned}
& \text { Person }(? p) \wedge \text { hasCensusProperty }(? p, ? \text { cprop }) \wedge \\
& \text { swrlb : equal }(? \text { cprop, specificProperty }) \wedge \\
& \text { Preference }(? p r) \wedge \text { hasPreference }(? p, ? \text { pr }) \\
& \Rightarrow \text { Person }(? p) \wedge \text { hasValue }(? \text { pr }, \text { assigned_value })
\end{aligned}
$$


The rule states that if a person $p$ has a specific census property cprop, then it can be inferred that this person holds the value assigned_value for a preference $p r$. An example may look as follows: If a person $p$ has an age of 18-25 years (cprop), then it can be concluded that the person $p$ has a preference for organic food $p r$ of 5. Assuming that $p r$ is for example measured on a Likert scale from 1 to 5 [30]. For reasons of comprehensibility, a simple example rule has been formulated. In practical modelling, preferences should be inferred using probability distributions as even within the age group of 18-25, there are various types of travellers with varying preferences. Moreover, the same preference $p r$ can be inferred from different census properties. The multiple inference of values for the same preference $p r$ results in probabilities for all attributes of the Likert scale that can be considered in final decision making. With our approach, researchers that are looking to customise the simulation model for research question specific purposes no longer have to deal with complex programming, but instead can make use of the benefits of semantic modelling. Using tangible persona models, settings for different agent types can be captured in a comprehensible form. Agents are assigned to persona types and are mirrored as individuals into the ontology. This means that individual conclusions can be drawn for the particular agent, and the inferred preferences can be incorporated into the decision-making behaviour of the agent.

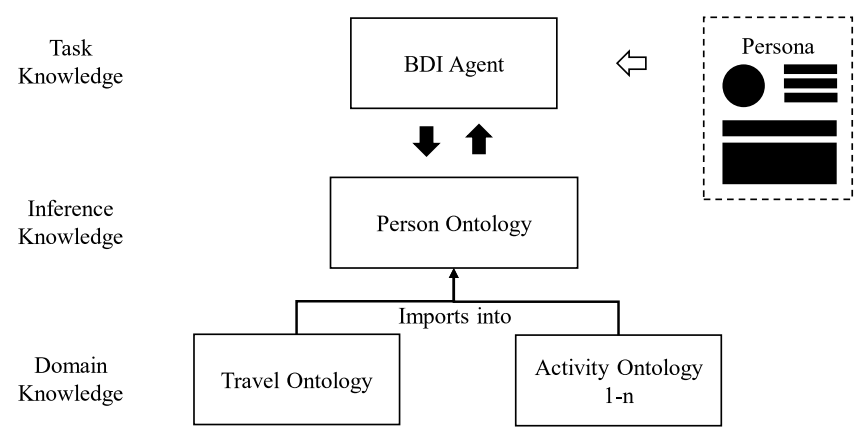

Fig. 2. Modelling Concept.

Finally for implementing task knowledge, the BDI model as a well established paradigm for implementing intelligent agents is particularly suitable. It enables software agents to perform action decisions (intentions) on the basis of defined goals (desires) and their modelled knowledge of their external world (beliefs) [31]. The BDI model is well suited to model actors in traffic scenarios: not only destinations of journeys but also optimisation goals e.g. minimal travel time, minimal emissions can be formulated as desires. Travel preferences and other parameters are potential beliefs that can be used to determine e.g. the selection of means of transportation. In our own previous work, we have given proof that a separation of general agent activity logic from aspects of modelling agent knowledge is an efficient and effective approach [32].

\section{PROOF OF CONCEPT}

To demonstrate the benefits of our proposed modelling method we have selected two example scenarios that fundamentally differ in types of mobility. In practical application this will be the case when specific research questions at hand deviate from the main focus of available simulators. We use the proposed modelling method and perform simulation as proof of concept. For both simulations we have exported geographical map data from OpenStreetMap [33] for the area around the city of Wetzlar which is located in Hesse, Germany, in addition we use data provided by the German census of 2011 [34]. Regarding the different types of travellers, for both scenarios, we created 12 persona based on a classification provided in [35] (see figure 3). The classification is based on various stages in life (age/occupation status) as well as family status and social strata/income (as illustrated in [36]) in order to represent the most significant groups of people in the German demographic. AGADE Traffic provides an option to create this type of persona using the web frontend.

For the first simulation, a commuter scenario has been modelled in which individuals start from various residential areas with all having the same target location. In real world scenarios, this is the case for example, when large gatherings take place or a large number of persons is commuting to the same workplace. We have marked the event arena in Wetzlar as the venue and thus, the desired target location for all agents. Furthermore, markers for each of the residential areas in the surrounding area of Wetzlar have been defined. The distribution of traveller agents starting from each residential area is based on data provided by the German census of 2011. A commuter scenario of this type primarily deals with knowledge about the traffic domain. In this context, route choice problems are commonly studied to determine current effects on the infrastructure or immediate surroundings. For example, research on transportation usually attempts to relieve particularly crowded road sections by improving traffic management, which is supposed to evenly distribute travel volume across alternative routes. For simulating this type of route choice problems, AGADE Traffic provides a default simulation model. The default simulation model generally assumes that all agents are travelling by car and performs routing based on the $\mathrm{A}^{*}$ algorithm (see [37]) that uses a cost functions based on shortest distance and additional geographical information. However in this context, the question of mode choice, e.g. travelling by car, bicycle, or walking, is just as relevant. Therefore, we perform customisation to the supplied default simulation model, just as researchers would like to do with research specific problems. The authors created an example ontology using OWL for modelling domain knowledge on traffic concepts. Using the ontology, agents obtain knowledge about different transportation modes available to them. For ease of exposition, in this paper we limit this ontol- 


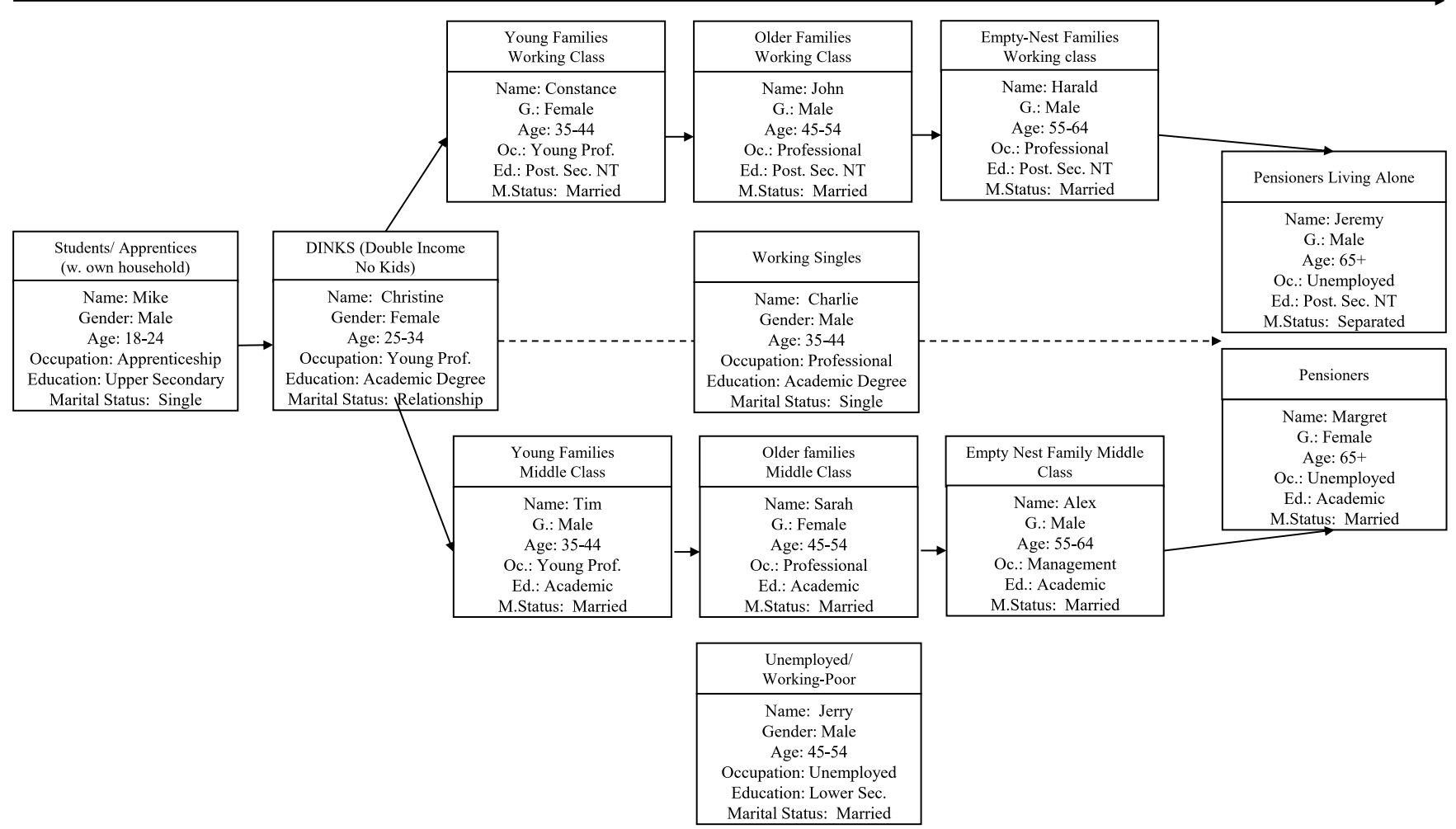

Fig. 3. Persona Models.

ogy to the concepts of various travel mode options. In particular, information on cars, bicycles, walking and public transport has been modelled. For more complex scenarios that require an expanded knowledge of the traffic domain, such as the simulation of Intelligent Transportation Systems (ITS) or testing of traffic light algorithms, this ontology can be extended. According to the modelling structure illustrated in figure 2, this ontology is equivalent to the travel ontology. Within this simulation, no activity ontology has been implemented as domain knowledge about traffic concepts is sufficient. In addition, we have extended the person ontology to define rules that reflect the decision-making behaviour regarding travel mode choice. The rules are created using semantic methods and do not require complex programming capabilities. The person ontology describes the traveller agent as a person concept which is itself described by various census properties. Furthermore, preferences are modelled in the person ontology which can be included as criteria for decision making. Using survey data, rules can be formulated that infer real values for the preferences of the agents based on the census properties defined in the respective personas. For this simulation, we have used data from [38]. For the integration of the inferred knowledge into the layer of Task Knowledge, it is not possible to completely avoid programming. Using our proposed modelling structure we have reduced the amount of programming required to the minimum. AGADE Traffic is written in Java and implements BDI agents using the JADEX framework [39]. For customisation purposes, AGADE Traffic makes use of the advantages of object oriented programming, and provides a central interface within the agent to implement decision-making algorithms or cost functions based on the inferred decision criteria from the ontology. For selection of travel mode, we have implemented a simple utility function that determines a personal utility score for each agent and mode based on utility values of the mode for various dimensions (monetary costs, eco-friendliness, etc.) and the inferred personal preference: Assuming $I$ being the set of modelled preferences in the ontology with $i \in I, n$ being the number of preferences in $I, U_{i}(m)$ being the utility value of a transportation mode $m$ for preference dimension $i$, and $p_{i}$ the inferred value of the personal preference of dimension $i$ for an agent. Based on this, we define UtilityScore $(m)=\sum_{i=0}^{n} U_{i}(m) * p_{i}$. Furthermore, we implemented mode selection based on Max(UtilityScore). This concludes the customisation performed for the first simulation scenario.

We have created a second scenario in which we simulate mobility related to grocery shopping. The characteristics of this scenario differ significantly from the first simulation. While all agents in the first simulation had a common target location, the grocery shopping scenario features different shopping locations that agents can travel to. Agents are assigned a generated list of food items to purchase and are then required to make decisions about the selection of supermarkets as well as mode of travel. It should be noted that supermarkets not only differ in product supply, but also 
available stock may vary in product quality and sustainability. Consequently, in some cases agents will not be able to purchase all items on the assigned grocery list at a selected grocery store, which requires them to visit subsequent target locations. In comparison to the first simulation, the decision-making process and the number of decision criteria involved are much more diverse. Using our proposed modelling structure, we demonstrate necessary customisation.

With regard to the difference in agent decision-making, it can be noted that agents have to decide on two major aspects; firstly, the selection of target locations (supermarkets) and secondly the selection of the travel mode. Decision criteria includes preferences not only regarding travel related aspects but also food related properties. Therefore, domain knowledge has to be extended by a separate ontology that provides information on various types of food and grocery stores, as well as information on available product inventory and further product related properties such as quality, sustainability, price tendency, etc. Considering the proposed modelling structure illustrated in Figure 2, this food ontology matches an activity ontology that researchers have to append when customising the provided default model for research specific scenarios. For this simulation, we thus make use of the same travel ontology from the first scenario, but append a new food ontology to the domain knowledge. We then extended the person ontology by rules that conclude information on food preferences. For this, we make use of polling data provided by [40]. With this, it is possible to infer all necessary preference information regarding both travel and food related aspects. Finally, we can use the provided programming interface within the agent to implement algorithms regarding decision-making of agents. The selection of supermarkets can for example be implemented in a similar manner using utility functions as demonstrated for travel mode selection. Given that the focus of this example is the description of the customisation process, at this point we will not further elaborate on the precise algorithm that we have implemented for this scenario. However, we will make source code and simulation data available. ${ }^{1}$ The algorithms for the implementation of the decision behaviour can be kept arbitrarily complex or simple depending on the research question at hand. With our proposed modelling structure, we create the basis for capturing all necessary decision preferences without complex programming and at the same time allow for flexible and adaptive scaling of the domain knowledge.

\section{CONCLUSION AND FUTURE WORK}

As customisation options in available traffic simulators are either simplified to aggregated parameters or associated with complex programming, existing simulation models have not been reused to their full potential. As a result, researchers dealing with specific research questions have rarely made used of available simulators, but instead created their own simulation environment

\footnotetext{
${ }^{1}$ see https://github.com/kite-cloud/agade-traffic
}

from scratch. Based on the ideas of the CommonKADS project and application of persona models and semantic methods, we have created a modelling structure that facilitates easy reuse by reducing required programming to the necessary minimum. Moreover, our modelling structure allows for adaptable modelling of agent knowledge as well as decision behaviour. For future work, modelling of both travel and activity related knowledge can be expanded. The creation and combination of further activity models for various domains may result in an open source library of activity knowledge that can be flexibly integrated, reused and customised for modelling complex research specific simulations.

\section{ACKNOWLEDGEMENT}

This research has been supported by a grant from the Karl-Vossloh-Stiftung (Project Number S0047/10053/2019).

\section{REFERENCES}

[1] M. Poeck and D. Zumkeller, "Die anwendung einer massnahmenempfindlichen prognosemethode am beispiel des grossraums nürnberg," in DVWG-Workshop Policy Sensitive Models, Giessen, 1976.

[2] K. Axhausen and R. Herz, "Simulating activity chains: German approach," Journal of Transportation Engineering, vol. 115, pp. 316-325, may 1989.

[3] Texas Transportation Institute, "Early deployment of transims: Issue paper," 1999.

[4] D. Krajzewicz, G. Hertkorn, C. Rössel, and P. Wagner, "Sumo (simulation of urban mobility)-an open-source traffic simulation," in Proceedings of the 4th middle East Symposium on Simulation and Modelling (MESM20002), pp. 183$187,2002$.

[5] A. Horni, K. Nagel, and K. Axhausen, The Multi-Agent Transport Simulation MATSim. Ubiquity Press, aug 2016.

[6] J. Auld, M. Hope, H. Ley, V. Sokolov, B. Xu, and K. Zhang, "POLARIS: Agent-based modeling framework development and implementation for integrated travel demand and network and operations simulations," Transportation Research Part C: Emerging Technologies, vol. 64, pp. 101-116, mar 2016.

[7] M. Jakob and Z. Moler, "Modular framework for simulation modelling of interaction-rich transport systems," in 16th International IEEE Conference on Intelligent Transportation Systems (ITSC 2013), pp. 2152-2159, IEEE, IEEE, oct 2013.

[8] M. Treiber and A. Kesting, "An open-source microscopic traffic simulator," IEEE Intelligent Transportation Systems Magazine, vol. 2, no. 3, pp. 6-13, 2010.

[9] S. Cheng and T. Nguyen, "TaxiSim: A multiagent simulation platform for evaluating taxi fleet operations," in Advanced Agent Technology, vol. 2, pp. 359-360, Springer Berlin Heidelberg, 2012.

[10] M. Horn, "Multi-modal and demand-responsive passenger transport systems: a modelling framework with embedded control systems," Transportation Research Part A: Policy and Practice, vol. 36, pp. 167-188, feb 2002.

[11] B. De Win, F. Piessens, W. Joosen, and T. Verhanneman, "On the importance of the separation-of-concerns principle in secure software engineering," in Workshop on the Application of Engineering Principles to System Security Design, pp. 1-10, 2002.

[12] D. McGuinness, F. Van Harmelen, et al., "Owl web ontology language overview," W3C recommendation, vol. 10, no. 10, p. 2004, 2004.

[13] I. Horrocks, P. Patel-Schneider, H. Boley, S. Tabet, B. Grosof, M. Dean, et al., "Swrl: A semantic web rule language combining owl and ruleml," W3C Member submission, vol. 21, no. 79, pp. 1-31, 2004.

[14] J. Geyer, J. Nguyen, T. Farrenkopf, and M. Guckert, "AGADE traffic 2.0 - a knowledge-based approach for multiagent traffic simulations," in Advances in Practical Appli- 
cations of Agents, Multi-Agent Systems, and Trustworthiness. The PAAMS Collection, pp. 417-420, Springer International Publishing, 2020.

[15] S. Blomkvist, "The user as a personality: A reflection on the theoritical and practical use of personas in hci design," Proceedings of the Technical report, pp. 1-13, 2006.

[16] A. Cooper, "The inmates are running the asylum. indianapolis, ia: Sams," Macmillan, 1999.

[17] S. Calde, K. Goodwin, and R. Reimann, "SHS orcas," in Case studies of the CHI2002|AIGA Experience Design FORUM on - CHI '02, pp. 2-16, ACM Press, 2002.

[18] N. Guarino, D. Oberle, and S. Staab, "What is an ontology?," in Handbook on Ontologies, pp. 1-17, Springer Berlin Heidelberg, 2009.

[19] A. Bazzan, M. do Amarante, T. Sommer, and A. Benavides, "Itsumo: an agent-based simulator for its applications," in Proc. of the 4th Workshop on Artificial Transportation Systems and Simulation. IEEE, p. 8, 2010.

[20] B. Torabi, M. Al-Zinati, and R. Wenkstern, "MATISSE 3.0: A large-scale multi-agent simulation system for intelligent transportation systems," in Advances in Practical Applications of Agents, Multi-Agent Systems, and Complexity: The PAAMS Collection, pp. 357-360, Springer International Publishing, 2018.

[21] M. Adnan, F. Pereira, C. Azevedo, K. Basak, M. Lovric, S. Raveau, Y. Zhu, J. Ferreira, C. Zegras, and M. BenAkiva, "Simmobility: A multi-scale integrated agent-based simulation platform," in 95th Annual Meeting of the Transportation Research Board Forthcoming in Transportation Research Record, 2016.

[22] V. Chu, J. Görmer, and J. Müller, "Atsim: Combining aimsum and jade for agent-based traffic simulation," in Proceedings of the 14th Conference of the Spanish Association for Artificial Intelligence (CAEPIA), vol. 1, 2011.

[23] S. Thulasidasan, S. Kasiviswanathan, S. Eidenbenz, E. Galli, S. Mniszewski, and P. Romero, "Designing systems for large-scale, discrete-event simulations: Experiences with the fasttrans parallel microsimulator," in 2009 International Conference on High Performance Computing (HiPC), pp. 428-437, IEEE, IEEE, dec 2009.

[24] M. Jakob, Z. Moler, A. Komenda, Z. Yin, A. Jiang, M. Johnson, M. Pěchouček, and M. Tambe, "Agentpolis: towards a platform for fully agent-based modeling of multimodal transportation," in Proceedings of the 11th International Conference on Autonomous Agents and Multiagent Systems-Volume 3, pp. 1501-1502, International Foundation for Autonomous Agents and Multiagent Systems, 2012.

[25] L. Quadrifoglio, M. Dessouky, and F. Ordóñez, "A simulation study of demand responsive transit system design," Transportation Research Part A: Policy and Practice, vol. 42 , pp. 718-737, may 2008.

[26] M. Wooldridge, "Agent-based software engineering," IEE Proceedings - Software Engineering, vol. 144, no. 1, p. 26, 1997.

[27] M. Guckert and T. Péus, "Problemlösungsmethoden reloaded: Integration von domänenwissen zur anwendung allgemeiner lösungsstrategien," Integration und Konnexion, p. $70,2013$.

[28] W. Clancey, Classification problem solving. Stanford University Stanford, CA, 1984.

[29] G. Schreiber, H. Akkermans, A. Anjewierden, N. Shadbolt, R. de Hoog, W. Van de Velde, R. Nigel, B. Wielinga, et al., Knowledge engineering and management: the CommonKADS methodology. MIT press, 2000.

[30] R. Likert, "A technique for the measurement of attitudes.," Archives of psychology, 1932.

[31] M. Bratman, D. Israel, and M. Pollack, "Plans and resourcebounded practical reasoning," Computational Intelligence, vol. 4, pp. 349-355, sep 1988.

[32] T. Farrenkopf, M. Guckert, N. Urquhart, and S. Wells, "Ontology based business simulations," Journal of Artificial Societies and Social Simulation, vol. 19, no. 4, 2016.

[33] M. Haklay and P. Weber, "OpenStreetMap: User-generated street maps," IEEE Pervasive Computing, vol. 7, pp. 12-18, oct 2008 .

[34] Statistische Ämter des Bundes und der Länder, Zensus 2011: Methoden und Verfahren. Wiesbaden, Hesse, Germany: Statistisches Bundesamt, 2015.

[35] GfK Consumer Panels, Consumers' choice '17 - neue Muster in der Ernährung: die Verbindung von Genuss, Gesundheit und Gemeinschaft in einer beschleunigten Welt eine Publikation anlässlich der Anuga 2017. GfK Consumer Panels and Bundesvereinigung der Deutschen Ernährungsindustrie e.V., 2017.

[36] N. Pestel and E. Sommer, "Analyse der verteilung von einkommen und vermögen in deutschland," tech. rep., Institute of Labor Economics (IZA), 2016.

[37] P. Hart, N. Nilsson, and B. Raphael, "A formal basis for the heuristic determination of minimum cost paths," IEEE Transactions on Systems Science and Cybernetics, vol. 4, no. 2, pp. 100-107, 1968 .

[38] U. Engel and M. Pötschke, "Mobilität und verkehrsmittelwahl 1999/2000." GESIS Datenarchiv, Köln. ZA4203 Datenfile Version 1.0.0, https://doi.org/10.4232/1.11591, 2013.

[39] L. Braubach, W. Lamersdorf, and A. Pokahr, "Jadex: Implementing a bdi-infrastructure for jade agents," 2003.

[40] Statista, "Lebensmittelkauf in deutschland," 2020.

\section{AUTHOR BIOGRAPHIES}

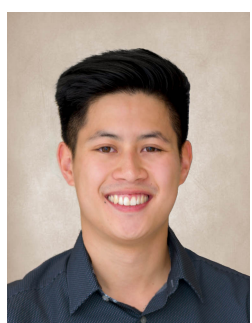

JOHANNES NGUYEN is a research assistant at Technische Hochschule Mittelhessen in Friedberg from which he received his master's degree. He is also a PhD student at Edinburgh Napier University. His research interests include multi-agent systems, mechanism design and semantic modelling particularly applied to the field of smart cities and future mobility.

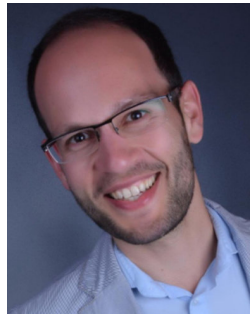

THOMAS FARRENKOPF is a lecturer at the at Technische Hochschule Mittelhessen. He completed a $\mathrm{PhD}$ degree in the School Of Computing at Edinburgh Napier University examining the use of software agents and ontologies for business simulation, applied to business games. His research areas include multi-agent systems, semantic modelling and computer science applied to industry projects.

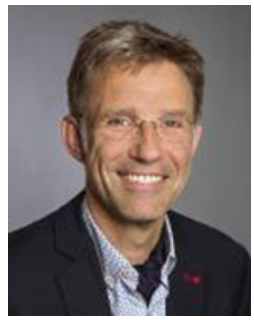

MICHAEL GUCKERT is a Professor of Applied Informatics at Technische Hochschule Mittelhessen and a head of department at Kompetenzzentrum für Informationstechnologie (KITE). He received a $\mathrm{PhD}$ in Computer Science from Philipps University Marburg. His research areas are multi-agent systems, model driven software development and applications of AI.

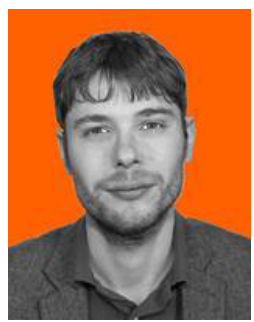

Simon T. Powers is a lecturer at Edinburgh Napier University. He gained his PhD from the University of Southampton, examining evolutionary explanations for cooperative social group formation. His research interests include computational social, political, and economic science in which he investigates the links between institutions, computer science, and multiagent systems.

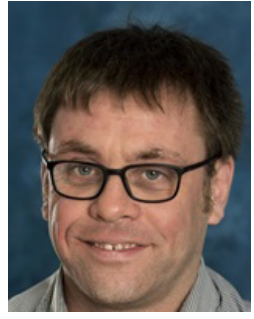

NEIL URQUHART is a lecturer at Edinburgh Napier University where he is Programme Leader for Computing Science. He gained his $\mathrm{PhD}$ from Edinburgh Napier University in 2002, examining the use of Software Agents and Evolutionary Algorithms to solve a real-world routing optimisation problem. His research interests include Evolutionary Computation and Agent-based Systems and their application to real-world problems. 